\title{
BOUNDS FOR THE RUIN PROBABILITY OF A DISCRETE-TIME RISK PROCESS
}

\author{
MAIKOL A. DIASPARRA, ${ }^{*}$ Universidad Simón Bolívar \\ ROSARIO ROMERA, ${ }^{* *}$ Universidad Carlos III de Madrid
}

\begin{abstract}
We consider a discrete-time risk process driven by proportional reinsurance and an interest rate process. We assume that the interest rate process behaves as a Markov chain. To reduce the risk of ruin, we may reinsure a part or even all of the reserve. Recursive and integral equations for ruin probabilities are given. Generalized Lundberg inequalities for the ruin probabilities are derived given a stationary policy. To illustrate these results, a numerical example is included.
\end{abstract}

Keywords: Risk process; ruin probability; Lundberg's inequality; proportional reinsurance

2000 Mathematics Subject Classification: Primary 91B30; 60K10

Secondary $60 \mathrm{~J} 05$

\section{Introduction}

In this paper we study the ruin probability in a generalized discrete-time risk process with a Markov chain interest model. Moreover, the risk model can be controlled by proportional reinsurance. Controlling a risk process is a very active area of research, particularly in the last decade; see, for instance, [6, pp. 2-21], [7], [9], and [10, pp. 1-26]. Nevertheless, obtaining explicit optimal solutions is a difficult task in a general setting. Hence, an alternative method commonly used in ruin theory is to derive inequalities or bounds for ruin probabilities (see [1, pp. 97-129], [4, pp. 1-32], [10, pp. 147-194], and [11, pp. 7-34]). Following Cai [2] and Cai and Dickson [3], we model the interest rate process as a denumerable state Markov chain. Inequalities for the ruin probabilities are derived by martingale and inductive techniques. The inequalities can be used to obtain upper bounds for the ruin probabilities. Our aim is to choose reinsurance control strategies to bound the ruin probability. For the sake of simplicity, we restrict ourselves to using stationary control policies.

The outline of the paper is as follows. In Section 2 the risk model is formulated. Some important special cases of this model are briefly discussed. In Section 3 we derive recursive equations for finite-horizon ruin probabilities and integral equations for the ultimate ruin probability. In Section 4 we obtain upper bounds for the ultimate probability of ruin. An analysis of the new bounds and a comparison with Lundberg's inequality is also included. Finally, in Section 5 we illustrate our results on the ruin probability in a risk process with a heavy tail claims distribution under proportional reinsurance and a Markov interest rate process. We conclude in Section 6 with some general comments and some suggestions for further research.

Received 28 August 2008; revision received 9 December 2008.

* Postal address: Department of Pure and Applied Mathematics, Universidad Simón Bolívar, 1080-A Caracas, Venezuela. Email address: maikold@yahoo.com

** Postal address: Department of Statistics, Universidad Carlos III de Madrid, 28903 Getafe, Spain.

Email address: mrromera@est-econ.uc3m.es 


\section{The model}

We consider a discrete-time insurance risk process in which the surplus $X_{n}$ varies according to the equation

$$
X_{n}=X_{n-1}\left(1+I_{n}\right)+\mathcal{C}\left(b_{n-1}\right)-h\left(b_{n-1}, Y_{n}\right) \text { for } n \geq 1
$$

with $X_{0}=x \geq 0$. Following Schmidli [10, p. 21] we introduce an absorbing (cemetery) state $\varkappa$, such that if $X_{n}<0$ or $X_{n}=\varkappa$ then $X_{n+1}=\varkappa$. We denote the state space by $\mathbb{X}=\mathbb{R} \cup \varkappa$. Let $Y_{n}$ be the total claims during the $n$th period (from time $n-1$ to time $n$ ), which we assume to form a sequence of independent and identically distributed random variables with common probability distribution function (PDF) $F$. The process can be controlled by reinsurance, that is, by choosing the retention level (or proportionality factor or risk exposure) $b \in \mathcal{B}$ of a reinsurance contract for one period, where $\mathcal{B}:=\left[b_{\min }, 1\right]$ and $b_{\min } \in(0,1]$ will be introduced below. Let $\left\{I_{n}\right\}_{n \geq 0}$ be the interest rate process; we suppose that $I_{n}$ evolves as a Markov chain with a denumerable (possibly finite) state space $\mathbb{I}$ consisting of nonnegative integers.

The function $h(b, y)$ with values in $[0, y]$ specifies the fraction of the claim $y$ paid by the insurer, and it also depends on the retention level $b$ at the beginning of the period. Hence, $y-h(b, y)$ is the part paid by the reinsurer. The retention level $b=1$ stands for the control action no reinsurance. In this paper we consider the case of proportional reinsurance, which means that

$$
h(b, y):=b y \quad \text { with retention level } b \in \mathcal{B} .
$$

The premium (income) rate $c$ is fixed. Since the insurer pays to the reinsurer a premium rate, which depends on the retention level $b$, we denote by $\mathcal{C}(b)$ the premium left for the insurer if the retention level $b$ is chosen, where

$$
0 \leq \mathcal{C}(b) \leq c, \quad b \in \mathcal{B}
$$

We define $b_{\min }:=\min \{b \in(0,1] \mid \mathcal{C}(b) \geq 0\}$. Moreover, $\mathcal{C}(b)$ is an increasing function that we will calculate according to the expected value principle with added safety loading $\theta$ from the reinsurer:

$$
\mathcal{C}(b)=c-(1+\theta)(1-b) \mathrm{E}[Y],
$$

where $Y$ is a generic random variable with PDF $F$.

We consider Markovian control policies $\pi=\left\{a_{n}\right\}_{n \geq 1}$, which at each time $n$ depend only on the current state, that is, $a_{n}\left(X_{n}\right):=b_{n}$ for $n \geq 0$. Abusing the notation, we will identify functions $a: \mathbb{X} \rightarrow \mathscr{B}$ with stationary strategies, where $\mathcal{B}=\left[b_{\min }, 1\right]$, the decision space. Consider an arbitrary initial state $X_{0}=x \geq 0$ (note that the initial value is not stochastic) and a control policy $\pi=\left\{a_{n}\right\}_{n \geq 1}$. Then, by iteration of (1), and assuming that (2) and (3) hold, it follows that, for $n \geq 1, X_{n}$ satisfies

$$
X_{n}=x \prod_{l=1}^{n}\left(1+I_{l}\right)+\sum_{l=1}^{n}\left(\mathcal{C}\left(b_{l-1}\right)-b_{l-1} Y_{l} \prod_{m=l+1}^{n}\left(1+I_{m}\right)\right) .
$$

Let $\left(p_{i j}\right)$ be the matrix of transition probabilities of $\left\{I_{n}\right\}$, i.e.

$$
p_{i j}:=\mathrm{P}\left(I_{n+1}=j \mid I_{n}=i\right)
$$


where $p_{i j} \geq 0$ and $\sum_{j} p_{i j}=1$ for all $i, j \in \mathbb{I}$. The ruin probability when using the policy $\pi$, given the initial surplus $x$, and the initial interest rate $I_{0}=i$ is defined as

$$
\psi^{\pi}(x, i):=\mathrm{P}^{\pi}\left(\bigcup_{k=1}^{\infty}\left\{X_{k}<0\right\} \mid X_{0}=x, I_{0}=i\right),
$$

which we can also express as

$$
\psi^{\pi}(x, i)=\mathrm{P}^{\pi}\left(X_{k}<0 \text { for some } k \geq 1 \mid X_{0}=x, I_{0}=i\right) .
$$

Similarly, the ruin probabilities in the finite-horizon case are given by

$$
\psi_{n}^{\pi}(x, i):=\mathrm{P}^{\pi}\left(\bigcup_{k=1}^{n}\left\{X_{k}<0\right\} \mid X_{0}=x, I_{0}=i\right) .
$$

Thus,

$$
\psi_{1}^{\pi}(x, i) \leq \psi_{2}^{\pi}(x, i) \leq \cdots \leq \psi_{n}^{\pi}(x, i) \leq \cdots
$$

and

$$
\lim _{n \rightarrow \infty} \psi_{n}^{\pi}(x, i)=\psi^{\pi}(x, i) .
$$

The following lemma is used below to simplify some calculations.

Lemma 1. For any given policy $\pi$, there is a function $\psi^{\pi}(x)$ such that

$$
\psi^{\pi}(x, i) \leq \psi^{\pi}(x)
$$

for every initial state $x>0$ and initial interest rate $I_{0}=i$.

Proof. By (1) and (2), the risk model is given by

$$
X_{n}=X_{n-1}\left(1+I_{n}\right)+\mathcal{C}\left(b_{n-1}\right)-b_{n-1} Y_{n} .
$$

Since $I_{n} \geq 0$, we have

$$
X_{n}=X_{n-1}\left(1+I_{n}\right)+\mathcal{C}\left(b_{n-1}\right)-b_{n-1} Y_{n} \geq X_{n-1}+\mathcal{C}\left(b_{n-1}\right)-b_{n-1} Y_{n} .
$$

Define recursively

$$
\widetilde{X}_{n}:=\widetilde{X}_{n-1}+\mathcal{C}\left(b_{n-1}\right)-b_{n-1} Y_{n}
$$

with $X_{0}=\tilde{X}_{0}=x$. Hence, $X_{n} \geq \widetilde{X}_{n}$ for all $n \in \mathbb{N}$. Clearly, if $X_{n}<0$ then $\widetilde{X}_{n}<0$. Let

$$
\varepsilon_{1}:=\left\{\omega \in \Omega \mid \bigcup_{n=1}^{\infty}\left\{X_{n}(\omega)<0\right\}\right\} \quad \text { and } \quad \varepsilon_{2}:=\left\{\omega \in \Omega \mid \bigcup_{n=1}^{\infty}\left\{\widetilde{X}_{n}(\omega)<0\right\}\right\},
$$

and note that $\varepsilon_{1} \subset \mathcal{E}_{2}$. Therefore,

$$
\mathrm{P}^{\pi}\left(\bigcup_{n=1}^{\infty}\left\{X_{n}<0\right\} \mid I_{0}=i\right) \leq \mathrm{P}^{\pi}\left(\bigcup_{n=1}^{\infty}\left\{\tilde{X}_{n}<0\right\} \mid I_{0}=i\right),
$$

and since the $\widetilde{X}_{n}$ do not depend on $I_{n}$, we obtain, from (6),

$$
\psi^{\pi}(x, i)=\mathrm{P}^{\pi}\left(\bigcup_{n=1}^{\infty}\left\{X_{n}<0\right\} \mid I_{0}=i\right) \leq \mathrm{P}^{\pi}\left(\bigcup_{n=1}^{\infty}\left\{\tilde{X}_{n}<0\right\}\right)=: \psi^{\pi}(x) .
$$

This completes the proof. 
We denote by $\Pi$ the policy space. A control policy $\pi^{*}$ is said to be optimal if, for any initial values $\left(X_{0}, I_{0}\right)=(x, i)$, we have

$$
\psi^{\pi^{*}}(x, i) \leq \psi^{\pi}(x, i) \text { for all } \pi \in \Pi .
$$

Schmidli [10, pp. 9-26] and Schäl [9] showed the existence of an optimal control policy for some special cases of the risk model (1). However, even in these special cases, it is extremely difficult to obtain closed expressions for $\psi^{\pi^{*}}(x, i)$. We are thus led to consider bounds for the ruin probabilities, which we do in Sections 3, 4, and 5, below. First, we note that (1) includes some interesting ruin models.

\subsection{Special cases}

To conclude this section we note the following subcases of the risk model (1).

- If $I_{n}=0$ and $b_{n}=1$ for all $n \geq 1$, then (1) reduces to the classical discrete-time risk model without investment and reinsurance:

$$
X_{k}=x-\sum_{t=1}^{k}\left(Y_{t}-c\right) .
$$

This is the well-known Cramér-Lundberg model, for which there are several bounds for the ruin probability [1, pp. 69-79], [4, pp. 1-8], [11, pp. 37-51].

- If $I_{n}=0$ and $b_{n} \in \mathscr{B}$ for $n \geq 1$, then the risk model (1) reduces to the discrete-time risk model with proportional reinsurance:

$$
X_{k}=x-\sum_{t=1}^{k}\left(b_{t-1} Y_{t}-\mathcal{C}\left(b_{t-1}\right)\right)
$$

Let $\psi^{\pi}(x):=\mathrm{P}^{\pi}\left(\bigcup_{k=1}^{\infty}\left\{X_{k}<0\right\} \mid X_{0}=x\right)$ be the corresponding ruin probability. More explicitly, by (9),

$$
\psi^{\pi}(x)=\mathrm{P}^{\pi}\left(\bigcup_{k=1}^{\infty}\left\{\sum_{t=1}^{k}\left[b_{t-1} Y_{t}-\mathcal{C}\left(b_{t-1}\right)\right]>x\right\} \mid X_{0}=x\right) .
$$

If we assume constant stationary strategies, say $b_{n}=b_{0}$ for all $n \geq 1$, and, in addition, that $\mathrm{E}\left[b_{0} Y\right]<\mathcal{C}\left(b_{0}\right)$, then there exists a constant $R_{0}>0$ satisfying

$$
\exp \left(-R_{0} \mathcal{C}\left(b_{0}\right)\right) \mathrm{E}\left[\exp \left(R_{0}\left(b_{0} Y\right)\right)\right]=1 .
$$

Therefore, by the classical Lundberg inequality for ruin probabilities (see [1], [4], and [11]),

$$
\psi^{\pi}(x) \leq \exp \left(-R_{0} x\right) \text { for } x \geq 0 .
$$

- Let $d_{n}$ be the constant, short-term dividend rate in the $n$th period (the dividends are payments made by a corporation to its shareholder members). Then the discrete-time risk model with stochastic interest rate and dividends is given by

$$
X_{n}=X_{n-1}\left(1+I_{n}\right)+\mathcal{C}\left(b_{n-1}\right)-h\left(b_{n-1}, Y_{n}\right)-d_{n} X_{n},
$$


where $h(b, y)$ is as in (2). Thus, rearranging terms,

$$
X_{n}=X_{n-1}\left(\frac{1+I_{n}}{1+d_{n}}\right)+\frac{\mathcal{C}\left(b_{n-1}\right)}{\left(1+d_{n}\right)}-\frac{h\left(b_{n-1}, Y_{n}\right)}{\left(1+d_{n}\right)} .
$$

Let $Y_{n}^{\prime}:=Y_{n} /\left(1+d_{n}\right)$ and $I_{n}{ }^{\prime}:=\left(I_{n}-d_{n}\right) /\left(1+d_{n}\right)$. Since $\left\{I_{n}\right\}$ and $\left\{Y_{n}\right\}$ are independent, then so are $\left\{I_{n}{ }^{\prime}\right\}$ and $\left\{Y_{n}^{\prime}\right\}$. Let $\mathcal{C}^{\prime}\left(b_{n-1}\right):=\mathcal{C}\left(b_{n-1}\right) /\left(1+d_{n}\right)$. Then the model becomes

$$
X_{n}=X_{n-1}\left(1+I_{n}{ }^{\prime}\right)+\mathfrak{C}^{\prime}\left(b_{n-1}\right)-h\left(b_{n-1}, Y_{n}^{\prime}\right),
$$

which from a statistical viewpoint is essentially the same as the model without dividends (1) and can be analyzed in a similar way.

- As an extension of the latter case, some companies have dividend reinvestment plans. These plans allow shareholders to use dividends to systematically buy small amounts of stock. Let $\widetilde{d}_{n}$ be the short-term dividend reinvestment rate in the $n$th period, $\widetilde{d}_{n} \in[0,1)$. Then, the discrete-time risk model with stochastic interest rate and dividends reinvestment is given by

$$
X_{n}=X_{n-1}\left(1+I_{n}\right)+\mathcal{C}\left(b_{n-1}\right)-h\left(b_{n-1}, Y_{n}\right)+\widetilde{d}_{n} X_{n}
$$

Hence, rearranging terms, we obtain

$$
X_{n}=X_{n-1}\left(\frac{1+I_{n}}{1-\widetilde{d}_{n}}\right)+\frac{\mathcal{C}\left(b_{n-1}\right)}{\left(1-\widetilde{d}_{n}\right)}-\frac{h\left(b_{n-1}, Y_{n}\right)}{\left(1-\widetilde{d}_{n}\right)}
$$

Let $Y_{n}^{\prime \prime}:=Y_{n} /\left(1-\widetilde{d}_{n}\right), I_{n}^{\prime \prime}:=\left(I_{n}-\widetilde{d}_{n}\right) /\left(1-\widetilde{d}_{n}\right)$, and $\mathcal{C}^{\prime \prime}\left(b_{n-1}\right):=\mathcal{C}\left(b_{n-1}\right) /\left(1-\widetilde{d}_{n}\right)$. It follows that

$$
X_{n}=X_{n-1}\left(1+I_{n}{ }^{\prime \prime}\right)+\mathcal{C}^{\prime \prime}\left(b_{n-1}\right)-h\left(b_{n-1}, Y_{n}^{\prime \prime}\right),
$$

which, again, is essentially the same as model (1).

Let us go back to the original risk model (1). Since determining ruin probabilities is essentially an infinite-horizon problem, it suffices to consider stationary strategies [10, pp. 154199]. In the next section we will derive recursive equations for the ruin probabilities and integral equations for the ultimate ruin probability associated to model (1).

Remark 1. Given a PDF $G$, we denote the tail of $G$ by $\bar{G}$, that is, $\bar{G}(x):=1-G(x)$.

\section{Recursive and integral equations for ruin probabilities}

In this section we first derive a recursive equation for $\psi_{n}^{\pi}(x, i)$. Secondly, we give an integral equation for $\psi^{\pi}(x, i)$. Finally, we obtain an equation for the ruin probability with horizon $n=1$ given $I_{0}=i, X_{0}=x$, and a stationary policy $\pi$. These results, which are valid for any initial interest rate, are summarized in the following lemma.

Lemma 2. Let $u(y):=b_{0} y-\mathcal{C}\left(b_{0}\right)$, where $b_{0}$ is the initial retention level. Let $\tau_{j}:=(x(1+j)$ $\left.+\mathcal{C}\left(b_{0}\right)\right) / b_{0}$, let $X_{0}=x \geq 0$, and let $p_{i j}$ be as in (5). Then

$$
\psi_{1}^{\pi}(x, i)=\sum_{j \in \mathbb{I}} p_{i j} \bar{F}\left(\tau_{j}\right)
$$


and, for $n=1,2, \ldots$,

$$
\psi_{n+1}^{\pi}(x, i)=\sum_{j \in \mathbb{I}} p_{i j} \int_{0}^{\tau_{j}} \psi_{n}^{\pi}(x(1+j)-u(y), j) \mathrm{d} F(y)+\sum_{j \in \mathbb{I}} p_{i j} \bar{F}\left(\tau_{j}\right) .
$$

Moreover,

$$
\psi^{\pi}(x, i)=\sum_{j \in \mathbb{I}} p_{i j} \int_{0}^{\tau_{j}} \psi^{\pi}(x(1+j)-u(y), j) \mathrm{d} F(y)+\sum_{j \in \mathbb{I}} p_{i j} \bar{F}\left(\tau_{j}\right) .
$$

Proof. Let $U_{k}:=u\left(Y_{k}\right)=b_{0} Y_{k}-\mathcal{C}\left(b_{0}\right)$. Given $Y_{1}=y$, the control strategy $\pi$, and $I_{1}=j$, from (4) we have $U_{1}=u(y)$. Therefore,

$$
X_{1}=x\left(1+I_{1}\right)-U_{1}=h_{1}-u(y),
$$

where $h_{1}=x(1+j)$. Thus, if $u(y)>h_{1}$ then

$$
\mathrm{P}^{\pi}\left(X_{1}<0 \mid Y_{1}=y, I_{1}=j, X_{0}=x, I_{0}=i\right)=1 .
$$

This implies that, for $u(y)>h_{1}$,

$$
\mathrm{P}^{\pi}\left(\bigcup_{k=1}^{n+1}\left\{X_{k}<0\right\} \mid Y_{1}=y, I_{1}=j, X_{0}=x, I_{0}=i\right)=1,
$$

while if $0 \leq u(y) \leq h_{1}$ then

$$
\mathrm{P}^{\pi}\left(X_{1}<0 \mid Y_{1}=y, I_{1}=j, X_{0}=x, I_{0}=i\right)=0 .
$$

Let $\left\{\widetilde{Y}_{n}\right\}_{n \geq 1}$ and $\left\{\widetilde{I}_{n}\right\}_{n \geq 0}$ be independent copies of $\left\{Y_{n}\right\}_{n \geq 1}$ and $\left\{I_{n}\right\}_{n \geq 0}$, respectively. Let $\widetilde{U}_{k}:=$ $b_{0} \widetilde{Y}_{k}-\mathcal{C}\left(b_{0}\right)$. Thus, (4) and (16) yield, for $0 \leq u(y) \leq h_{1}$,

$$
\begin{aligned}
& \mathrm{P}^{\pi}\left(\bigcup_{k=1}^{n+1}\left\{X_{k}<0\right\} \mid Y_{1}=y, I_{1}=j, X_{0}=x, I_{0}=i\right) \\
& =\mathrm{P}^{\pi}\left(\bigcup_{k=2}^{n+1}\left\{X_{k}<0\right\} \mid Y_{1}=y, I_{1}=j, X_{0}=x, I_{0}=i\right) \\
& =\mathrm{P}^{\pi}\left(\bigcup_{k=2}^{n+1}\left\{\left(h_{1}-u(y)\right) \prod_{l=1}^{k}\left(1+I_{l}\right)-\sum_{l=1}^{k} U_{l} \prod_{m=l+1}^{k}\left(1+I_{m}\right)<0\right\} \mid X_{0}=x, I_{1}=j\right) \\
& =\mathrm{P}^{\pi}\left(\bigcup_{k=1}^{n}\left\{\left(h_{1}-u(y)\right) \prod_{l=1}^{k}\left(1+\widetilde{I}_{l}\right)-\sum_{l=1}^{k} \tilde{U}_{l} \prod_{m=l+1}^{k}\left(1+\widetilde{I}_{m}\right)<0\right\} \mid X_{0}=x, \tilde{I}_{0}=j\right) \\
& =\psi_{n}^{\pi}\left(h_{1}-u(y), j\right) \\
& =\psi_{n}^{\pi}(x(1+j)-u(y), j),
\end{aligned}
$$

where the second equality follows from the Markov property of $\left\{I_{n}\right\}_{n \geq 0}$, and the independence of $\left\{Y_{n}\right\}_{n \geq 1}$ and $\left\{I_{n}\right\}_{n \geq 0}$. 
Let us now consider the event $\mathcal{A}=\left\{Y_{1}=y, I_{1}=j, X_{0}=x, I_{0}=i\right\}$, and recall that $F(y)=\mathrm{P}(Y \leq y)$. From (4) and (7), we obtain

$$
\begin{aligned}
\psi_{n+1}^{\pi}(x, i) & =\mathrm{P}^{\pi}\left(\bigcup_{k=1}^{n+1}\left\{X_{k}<0\right\} \mid X_{0}=x, I_{0}=i\right) \\
& =\sum_{j \in \mathbb{I}} p_{i j} \int_{0}^{\infty} \mathrm{P}^{\pi}\left(\bigcup_{k=1}^{n+1}\left\{X_{k}<0\right\} \mid \mathcal{A}\right) \mathrm{d} F(y) .
\end{aligned}
$$

Then, recalling that $\tau_{j}=\left(x(1+j)+\mathcal{C}\left(b_{0}\right)\right) / b_{0}$,

$$
\begin{aligned}
\psi_{n+1}^{\pi}(x, i)= & \sum_{j \in \mathbb{I}} p_{i j}\left\{\int_{0}^{\tau_{j}} \mathrm{P}^{\pi}\left(\bigcup_{k=1}^{n+1}\left\{X_{k}<0\right\} \mid \mathcal{A}\right) \mathrm{d} F(y)\right. \\
& \left.+\int_{\tau_{j}}^{\infty} \mathrm{P}^{\pi}\left(\bigcup_{k=1}^{n+1}\left\{X_{k}<0\right\} \mid \mathcal{A}\right) \mathrm{d} F(y)\right\} \\
= & \sum_{j \in \mathbb{I}} p_{i j}\left\{\int_{0}^{\tau_{j}} \psi_{n}^{\pi}(x(1+j)-u(y), j) \mathrm{d} F(y)+\int_{\tau_{j}}^{\infty} \mathrm{d} F(y)\right\} \\
= & \sum_{j \in \mathbb{I}} p_{i j}\left\{\int_{0}^{\tau_{j}} \psi_{n}^{\pi}(x(1+j)-u(y), j) \mathrm{d} F(y)+\bar{F}\left(\tau_{j}\right)\right\} .
\end{aligned}
$$

This gives (13). In particular,

$$
\psi_{1}^{\pi}(x, i)=\sum_{j \in \mathbb{I}} p_{i j} \bar{F}\left(\tau_{j}\right)
$$

Finally, letting $n \rightarrow \infty$ in (17) and using dominated convergence, we obtain

$$
\lim _{n \rightarrow \infty} \psi_{n+1}^{\pi}(x, i)=\psi^{\pi}(x, i),
$$

and (14) follows.

Remark 2. If we consider the risk model without reinsurance, that is, $b=1$, we obtain similar results to those in [3].

\section{Bounds for ruin probabilities}

We will use the results obtained in Section 3 to find upper bounds for the ruin probabilities with an infinite horizon, taking into account the information contributed by the Markov chain of the interest rate process. We derive a functional for the ultimate ruin probability in terms of the new worse than used in convex ordering; see Remark 3, below. This idea was first introduced by Willmot and Lin [11] and has been generalized by other authors.

We will present two upper bounds for the ruin probabilities. The first bound is obtained by an inductive approach and the second by a martingale approach. These bounds are discussed in Remark 4, at the end of this section. 


\subsection{Bounds obtained by the inductive approach}

Theorem 1. Let $R_{0}>0$ be the constant satisfying (10). Then, for all $x \geq 0$ and $i \in \mathbb{I}$,

$$
\begin{aligned}
\psi^{\pi}(x, i) & \leq \beta \sum_{j \in \mathbb{I}} p_{i j} \mathrm{E}^{\pi}\left[\exp \left(-R_{0} x(1+j)\right)\right] \\
& =\beta \mathrm{E}^{\pi}\left[\exp \left(-R_{0}\left[x\left(1+I_{1}\right)\right]\right) \mid I_{0}=i\right],
\end{aligned}
$$

where $\beta \equiv \beta\left(b_{0}\right)$ and is given by

$$
\beta^{-1}=\inf _{t \geq 0} \frac{\int_{t}^{\infty} \exp \left(R_{0} b_{0} y\right) \mathrm{d} F(y)}{\exp \left(R_{0} b_{0} t\right) \bar{F}(t)} .
$$

Proof. It suffices to show that the rightmost term in (18) is an upper bound for $\psi_{n}^{\pi}(x, i)$ for all $n \geq 1$. We will prove this by induction. First note that

$$
\begin{aligned}
\bar{F}(\theta) & =\left(\frac{\int_{\theta}^{\infty} \exp \left(R_{0} b_{0} y\right) \mathrm{d} F(y)}{\exp \left(R_{0} b_{0} \theta\right) \bar{F}(\theta)}\right)^{-1} \exp \left(-R_{0} b_{0} \theta\right) \int_{\theta}^{\infty} \exp \left(R_{0} b_{0} y\right) \mathrm{d} F(y) \\
& \leq \beta \exp \left(-R_{0} b_{0} \theta\right) \int_{\theta}^{\infty} \exp \left(R_{0} b_{0} y\right) \mathrm{d} F(y) \\
& \leq \beta \exp \left(-R_{0} b_{0} \theta\right) \mathrm{E}^{\pi}\left[\exp \left(R_{0} b Y_{1}\right)\right] \quad \text { for any } \theta \geq 0 .
\end{aligned}
$$

This implies that, for every $x \geq 0, i \geq 0$, and $b_{0} \in \mathcal{B}$, by (12) and (19), we have

$$
\begin{aligned}
\psi_{1}^{\pi}(x, i) & =\sum_{j \in \mathbb{I}} p_{i j} \bar{F}\left(\tau_{j}\right) \\
& \leq \sum_{j \in \mathbb{I}} p_{i j}\left(\beta \mathrm{E}^{\pi}\left[\exp \left(R_{0} b Y_{1}\right)\right] \exp \left(-R_{0} b_{0}\left(\frac{x(1+j)+\mathcal{C}\left(b_{0}\right)}{b_{0}}\right)\right)\right) \\
& =\beta \mathrm{E}^{\pi}\left[\exp \left(R_{0} b Y_{1}\right)\right] \sum_{j \in \mathbb{I}} p_{i j} \exp \left(-R_{0}\left[x(1+j)+\mathcal{C}\left(b_{0}\right)\right]\right) \\
& =\beta \mathrm{E}^{\pi}\left[\exp \left(R_{0} b Y_{1}\right)\right] \mathrm{E}^{\pi}\left[\exp \left(-R_{0}\left[x\left(1+I_{1}\right)+\mathcal{C}(b)\right]\right) \mid I_{0}=i\right] \\
& =\beta \mathrm{E}^{\pi}\left[\exp \left(R_{0} b Y_{1}\right)\right] \mathrm{E}^{\pi}\left[\exp \left(-R_{0} \mathcal{C}(b)\right)\right] \mathrm{E}^{\pi}\left[\exp \left(-R_{0} x\left(1+I_{1}\right)\right) \mid I_{0}=i\right] \\
& =\beta \mathrm{E}^{\pi}\left[\exp \left(-R_{0}\left[\mathcal{C}(b)-b Y_{1}\right]\right)\right] \mathrm{E}^{\pi}\left[\exp \left(-R_{0} x\left(1+I_{1}\right)\right) \mid I_{0}=i\right] \\
& =\beta \mathrm{E}^{\pi}\left[\exp \left(-R_{0} x\left(1+I_{1}\right)\right) \mid I_{0}=i\right] \quad(\operatorname{by}(10)) .
\end{aligned}
$$

This shows that the desired result holds for $n=1$. To prove the result for general $n \geq 1$, the induction hypothesis is that, for some $n \geq 1$, and every $x \geq 0$ and $i \in \mathbb{I}$,

$$
\psi_{n}^{\pi}(x, i) \leq \beta \mathrm{E}^{\pi}\left[\exp \left(-R_{0} x\left(1+I_{1}\right)\right) \mid I_{0}=i\right] .
$$

Now, let $0 \leq y \leq \tau_{j}$, where $\tau_{j}$ is as defined in Lemma 2. Furthermore, in (20), replace $x$ and $i$ by $x(1+j)+\mathcal{C}\left(b_{0}\right)-b_{0} y$ and $j$, respectively, to obtain

$$
\begin{aligned}
\psi_{n}^{\pi}(x & \left.(1+j)+\mathcal{C}\left(b_{0}\right)-b_{0} y, j\right) \\
& \leq \beta \mathrm{E}^{\pi}\left[\exp \left(-R_{0}[x(1+j)+\mathcal{C}(b)-b y]\left(1+I_{1}\right)\right) \mid I_{0}=j\right] \\
& \leq \beta \exp \left(-R_{0}\left[x(1+j)+\mathcal{C}\left(b_{0}\right)-b_{0} y\right]\right) .
\end{aligned}
$$


Therefore, substituting (21) into (13), we obtain

$$
\begin{aligned}
\psi_{n+1}^{\pi}(x, i) \leq & \sum_{j \in \mathbb{I}} p_{i j}\left(\beta \exp \left(-R_{0}\left[x(1+j)+\mathcal{C}\left(b_{0}\right)\right]\right) \int_{\tau_{j}}^{\infty} \exp \left(R_{0} b_{0} y\right) \mathrm{d} F(y)\right) \\
& +\sum_{j \in \mathbb{I}} p_{i j}\left(\beta \exp \left(-R_{0}\left[x(1+j)+\mathcal{C}\left(b_{0}\right)\right]\right) \int_{0}^{\tau_{j}} \exp \left(R_{0} b_{0} y\right) \mathrm{d} F(y)\right) \\
= & \sum_{j \in \mathbb{I}} p_{i j}\left(\beta \exp \left(-R_{0}\left[x(1+j)+\mathcal{C}\left(b_{0}\right)\right]\right) \int_{0}^{\infty} \exp \left(R_{0} b_{0} y\right) \mathrm{d} F(y)\right) \\
= & \beta \mathrm{E}^{\pi}\left[\exp \left(R_{0} b Y_{1}\right)\right] \sum_{j \in \mathbb{I}} p_{i j} \exp \left(-R_{0}\left[x(1+j)+\mathcal{C}\left(b_{0}\right)\right]\right) \\
= & \beta \mathrm{E}^{\pi}\left[\exp \left(R_{0} b Y_{1}\right)\right] \mathrm{E}^{\pi}\left[\exp \left(-R_{0} \mathcal{C}(b)\right)\right] \mathrm{E}^{\pi}\left[\exp \left(-R_{0} x\left(1+I_{1}\right)\right) \mid I_{0}=i\right] \\
= & \beta \mathrm{E}^{\pi}\left[\exp \left(-R_{0} x\left(1+I_{1}\right)\right) \mid I_{0}=i\right] .
\end{aligned}
$$

Hence, (20) holds for any $n=1,2, \ldots$, and letting $n \rightarrow \infty$ in (20) we obtain (18). This completes the proof.

As an application of Theorem 1, we now consider the special case in which the claim distribution is in the class of NWUC distributions [11, p. 25], which are defined as follows.

Remark 3. A distribution $F$ concentrated on $(0, \infty)$ is said to be new worse than used in convex (NWUC) ordering if, for all $x, y \geq 0$,

$$
\int_{x+y}^{\infty} \bar{F}(z) \mathrm{d} z \geq \bar{F}(y) \int_{x}^{\infty} \bar{F}(z) \mathrm{d} z .
$$

Corollary 1. Under the hypotheses of Theorem 1, and assuming that $\mathrm{E}^{\pi}\left[\exp \left(R_{0} b Y_{1}\right)\right]<\infty$ for all $b \in \mathcal{B}$ and that, in addition, $F$ is an NWUC distribution, we have

$$
\psi^{\pi}(x, i) \leq\left(\mathrm{E}^{\pi}\left[\exp \left(R_{0} b Y_{1}\right)\right]\right)^{-1} \mathrm{E}^{\pi}\left[\exp \left(-R_{0} x\left(1+I_{1}\right)\right) \mid I_{0}=i\right] .
$$

Proof. Following Willmot and Lin [11, pp. 96-97], let $r:=R_{0} b>0$. Therefore,

$$
\beta^{-1}:=\inf _{t \geq 0} \frac{\int_{t}^{\infty} \mathrm{e}^{r y} \mathrm{~d} F(y)}{\mathrm{e}^{r t} \bar{F}(t)}=\int_{0}^{\infty} \mathrm{e}^{r y} \mathrm{~d} F(y),
$$

that is, $\beta^{-1}=\mathrm{E}^{\pi}\left[\exp \left(R_{0} b Y_{1}\right)\right]$. Finally, substituting this equality into (18), we obtain (22).

\subsection{Bounds by the martingale approach}

Another way of deriving upper bounds for ruin probabilities is the martingale approach. To this end, let $V_{n}:=X_{n} \prod_{l=1}^{n}\left(1+I_{l}\right)^{-1}$ with $n \geq 1$ be the so-called discounted risk process. The ruin probabilities $\psi_{n}^{\pi}$ in (7) associated to the process $\left\{V_{n}, n=1,2 \ldots\right\}$ are

$$
\psi_{n}^{\pi}(x, i)=\mathrm{P}^{\pi}\left(\bigcup_{k=1}^{n}\left(V_{k}<0\right) \mid X_{0}=x, I_{0}=i\right) .
$$

In the classical risk model, $\left\{\exp \left(-R_{0} X_{n}\right)\right\}_{n \geq 1}$ is a martingale. However, for our model (4), there is no constant $r>0$ such that $\left\{\exp \left(-r X_{n}\right)\right\}_{n \geq 1}$ is a martingale. Still, there exists a 
constant $r>0$ such that $\left\{\exp \left(-r V_{n}\right)\right\}_{n \geq 1}$ is a supermartingale, which allows us to derive probability inequalities by the optional stopping theorem. Such a constant is defined in the following proposition.

Proposition 1. Assume that $\mathrm{E}^{\pi}\left[\mathcal{C}(b)-b Y_{1}\right]>0$. In addition, we suppose that, for each $i \in \mathbb{I}$, there exists $\rho_{i}>0$ satisfying

$$
\mathrm{E}^{\pi}\left[\exp \left(-\rho_{i}\left[\mathcal{C}(b)-b Y_{1}\right]\left(1+I_{1}\right)^{-1}\right) \mid I_{0}=i\right]=1 .
$$

Then

$$
R_{1}:=\min _{i \in \mathbb{I}} \rho_{i} \geq R_{0},
$$

and, furthermore, for all $i \in \mathbb{I}$,

$$
\mathrm{E}^{\pi}\left[\exp \left(-R_{1}\left[\mathcal{C}(b)-b Y_{1}\right]\left(1+I_{1}\right)^{-1}\right) \mid I_{0}=i\right] \leq 1 .
$$

Proof. For each $i \in \mathbb{I}$, let

$$
l_{i}(r):=\mathrm{E}^{\pi}\left[\exp \left(-r[\mathcal{C}(b)-b Y]\left(1+I_{1}\right)^{-1}\right) \mid I_{0}=i\right]-1 \text { for } r>0 .
$$

Then the first derivative of $l_{i}(r)$ at $r=0$ is

$$
l_{i}^{\prime}(0)=\mathrm{E}^{\pi}[-(\mathcal{C}(b)-b Y)] \mathrm{E}^{\pi}\left[\left(1+I_{1}\right)^{-1} \mid I_{0}=i\right]<0 \quad \text { (by independence) }
$$

and the second derivative is

$$
l_{i}^{\prime \prime}(r)=\mathrm{E}^{\pi}\left[\left((\mathcal{C}(b)-b Y)\left(1+I_{1}\right)^{-1}\right)^{2} \exp \left(-r[\mathcal{C}(b)-b Y]\left(1+I_{1}\right)^{-1}\right) \mid I_{0}=i\right]>0 .
$$

This shows that $l_{i}(r)$ is a convex function. Let $\rho_{i}$ be the unique positive root of the equation $l_{i}(r)=0$ on $(0, \infty)$. Furthermore, if $0<\rho \leq \rho_{i}$ then $l_{i}(\rho) \leq 0$. However,

$$
\begin{aligned}
& \mathrm{E}^{\pi}\left[\exp \left(-R_{0}[\mathcal{C}(b)-b Y]\left(1+I_{1}\right)^{-1}\right) \mid I_{0}=i\right] \\
& \quad=\sum_{j \in \mathbb{I}} p_{i j} \mathrm{E}\left[\exp \left(-R_{0}\left[\mathcal{C}\left(b_{0}\right)-b_{0} Y\right](1+j)^{-1}\right)\right] \quad \text { (by Jensen's inequality) } \\
& \quad \leq \sum_{j \in \mathbb{I}} p_{i j} \mathrm{E}\left[\exp \left(-R_{0}\left[\mathcal{C}\left(b_{0}\right)-b_{0} Y_{1}\right]\right)\right]^{(1+j)^{-1}} .
\end{aligned}
$$

Consequently, by (10) we have $\mathrm{E}\left[\exp \left(-R_{0}\left[\mathcal{C}\left(b_{0}\right)-b_{0} Y_{1}\right]\right)\right]=1$. Hence, since $\sum_{j \in \mathbb{I}} p_{i j}=1$,

$$
\mathrm{E}^{\pi}\left[\exp \left(-R_{0}[\mathcal{C}(b)-b Y]\left(1+I_{1}\right)^{-1}\right) \mid I_{0}=i\right] \leq 1 .
$$

This implies that $l_{i}\left(R_{0}\right) \leq 0$. Moreover, $R_{0} \leq \rho_{i}$ for all $i$, and so

$$
R_{1}:=\min _{i \in \mathbb{I}} \rho_{i} \geq R_{0}
$$

Thus, (23) holds. In addition, $R_{1} \leq \rho_{i}$ for all $i \in \mathbb{I}$, which implies that $l_{i}\left(R_{1}\right) \leq 0$. This yields (24).

Theorem 2. Under the hypotheses of Proposition 1, for all $i \in \mathbb{I}$ and $x \geq 0$,

$$
\psi^{\pi}(x, i) \leq \exp \left(-R_{1} x\right) .
$$


Proof. By (4), the discounted risk process $V_{k}:=X_{k} \prod_{l=1}^{k}\left(1+I_{l}\right)^{-1}$ satisfies

$$
V_{k}:=x+\sum_{l=1}^{k}\left(\left(\mathcal{C}\left(b_{0}\right)-b_{0} Y_{l}\right) \prod_{t=1}^{l}\left(1+I_{t}\right)^{-1}\right) .
$$

Let $S_{n}=\exp \left(-R_{1} V_{n}\right)$. Then

$$
S_{n+1}=S_{n} \exp \left(-R_{1}\left(\mathcal{C}\left(b_{0}\right)-b_{0} Y_{n+1}\right) \prod_{t=1}^{n+1}\left(1+I_{t}\right)^{-1}\right) .
$$

Thus, for any $n \geq 1$,

$$
\begin{aligned}
\mathrm{E}^{\pi}[ & \left.S_{n+1} \mid Y_{1}, \ldots Y_{n}, I_{1}, \ldots I_{n}\right] \\
& =S_{n} \mathrm{E}\left[\exp \left(-R_{1}\left(\mathcal{C}\left(b_{0}\right)-b_{0} Y_{n+1}\right) \prod_{t=1}^{n+1}\left(1+I_{t}\right)^{-1}\right) \mid Y_{1}, \ldots Y_{n}, I_{1}, \ldots I_{n}\right] \\
& =S_{n} \mathrm{E}\left[\exp \left(-R_{1}\left(\mathcal{C}\left(b_{0}\right)-b_{0} Y_{n+1}\right)\left(1+I_{n+1}\right)^{-1} \prod_{t=1}^{n}\left(1+I_{t}\right)^{-1}\right) \mid I_{1}, \ldots I_{n}\right] \\
& \leq S_{n} \mathrm{E}\left(\left[\exp \left(-R_{1}\left(\mathcal{C}\left(b_{0}\right)-b_{0} Y_{n+1}\right)\left(1+I_{n+1}\right)^{-1}\right) \mid I_{1}, \ldots I_{n}\right]\right)^{\prod_{t=1}^{n}\left(1+I_{t}\right)^{-1}} \\
& \leq S_{n} .
\end{aligned}
$$

This implies that $\left\{S_{n}\right\}_{n \geq 1}$ is a supermartingale.

Let $T_{i}=\min \left\{n: V_{n}<0 \mid I_{0}=i\right\}$, where $V_{n}$ is given by (26). Then $T_{i}$ is a stopping time and $n \wedge T_{i}:=\min \left\{n, T_{i}\right\}$ is a finite stopping time. Thus, by the optional stopping theorem for martingales we obtain

$$
\mathrm{E}^{\pi}\left[S_{n \wedge T_{i}}\right] \leq \mathrm{E}^{\pi}\left[S_{0}\right]=\exp \left(-R_{1} x\right) .
$$

Hence,

$$
\begin{aligned}
\exp \left(-R_{1} x\right) & \geq \mathrm{E}^{\pi}\left[S_{n \wedge T_{i}}\right] \\
& \geq \mathrm{E}^{\pi}\left[\left(S_{n \wedge T_{i}}\right) \mathbf{1}_{\left\{T_{i} \leq n\right\}}\right] \\
& \geq \mathrm{E}^{\pi}\left[\left(S_{T_{i}}\right) \mathbf{1}_{\left\{T_{i} \leq n\right\}}\right] \\
& =\mathrm{E}^{\pi}\left[\exp \left(-R_{1} V_{T_{i}}\right) \mathbf{1}_{\left\{T_{i} \leq n\right\}}\right] \\
& \geq \mathrm{E}^{\pi}\left[\mathbf{1}_{\left\{T_{i} \leq n\right\}}\right] \\
& =\psi_{n}^{\pi}(x, i),
\end{aligned}
$$

where (28) follows because $V_{T_{i}}<0$. Thus, by letting $n \rightarrow \infty$ in (28) we obtain (25).

Remark 4. Summarizing, we have three upper bounds for the ruin probabilities with infinite horizon. First, the Lundberg bound, which depends only on $R_{0}$, the Lundberg exponential in (10) and (11). Second, the inductive bound (18), which depends on $R_{0}$ and also on the interest rate process. Third, the martingale bound in (25), which depends on $R_{1}$. Note that the last two bounds are sharper than the Lundberg bound. Observe also that the number of operations to obtain $R_{1}$ in (25) is higher than that to obtain $R_{0}$ in (18).

In the next section we present some numerical results. 


\section{Numerical results}

To illustrate the bounds obtained in Theorems 1 and 2, we present a numerical example that uses MATLAB ${ }^{\circledR}$ and MAPLE ${ }^{\circledR}$ implementations for different values of the retention level $b$.

We assume that the claim amount in year $k$ is $Y_{k}$, which has a gamma density $\operatorname{gamma}\left(\frac{1}{2}, 2\right)$. Since this distribution is NWUC, we will use (22).

The annual premium is $c=1.1$; namely, there is a loading of $10 \%$ given by the reinsurer. In this example,

$$
\mathcal{C}(b)=1.1-(1.1)(1-b)>0 \quad \text { if } \quad b \in(0,1] .
$$

Consider an interest model with three possible interest rates:

$$
\mathbb{I}=\{6 \%, 8 \%, 10 \%\} .
$$

The transition matrix (see (5)) is given by

$$
\left(\begin{array}{ccc}
0.2 & 0.8 & 0 \\
0.15 & 0.7 & 0.15 \\
0 & 0.8 & 0.2
\end{array}\right)
$$

Thus, our interest rate model incorporates mean reversion to a level of $8 \%$. In this example, $\mathcal{B}=(0,1]$.

In Figure 1 we show that the relation between $R_{l}$ and $b$, with $l \in\{0,1\}$, is inversely proportional. Remember that $b=1$ means no reinsurance, and so we will hope to have a small value of $R_{l}$. Analogously, when $b$ is close to 0 (reinsure almost everything), $R_{l}$ becomes extremely large.

The numerical results in Table 1 show that the upper bound in (18) can be tighter than that in (25). This suggests that the upper bounds derived by the inductive approach are tighter than the upper bounds obtained by supermartingales. In addition, Table 1 shows that the upper bounds derived in this paper are sharper than the Lundberg upper bound.

Remark 5. In the case in which $b=1$, our results are the same as in [3].
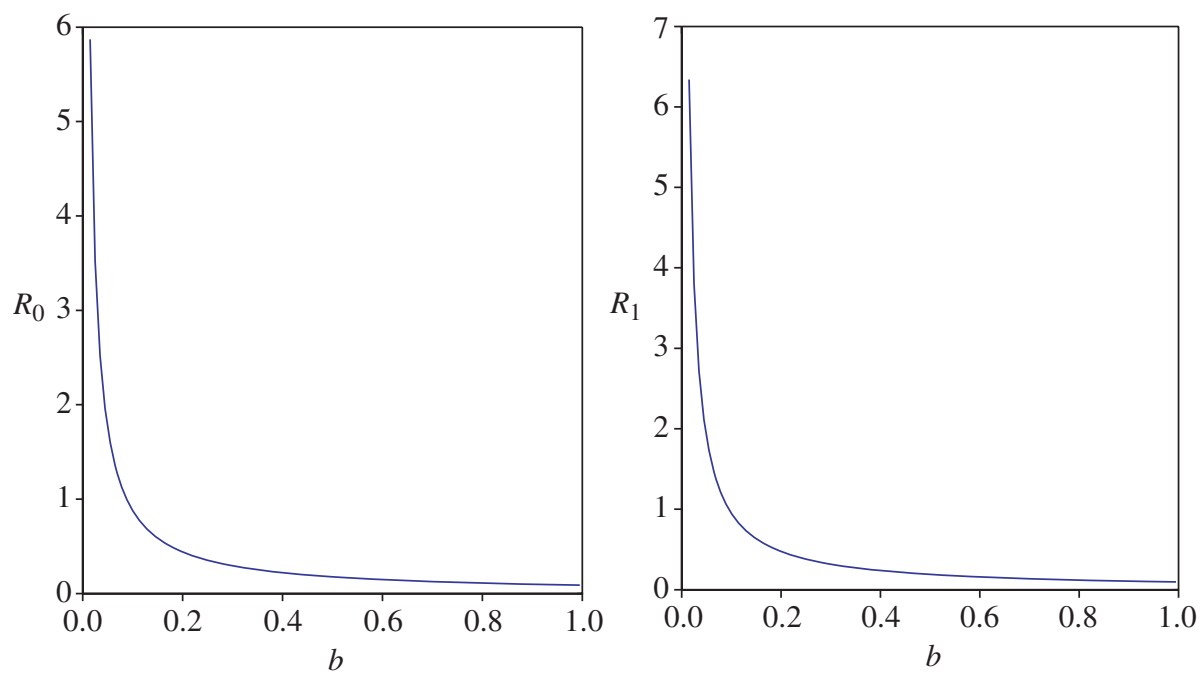

FIGURE 1: Left: the relation between $R_{0}$ and $b$. Right: the relation between $R_{1}$ and $b$. 
TABLE 1: Table of upper bounds for ruin probabilities with $x=5$ and $i=8 \%$.

\begin{tabular}{clllcc}
\hline Retention level $b$ & Lundberg & Induction & Martingale & $R_{0}$ & \multicolumn{1}{c}{$R_{1}$} \\
\hline 0.01 & $0.752 \times 10^{-19}$ & $0.226 \times 10^{-20}$ & $0.224 \times 10^{-20}$ & 8.8067 & 9.5091 \\
0.25 & 0.171 & 0.135 & 0.149 & 0.352 & 0.380 \\
0.50 & 0.414 & 0.350 & 0.386 & 0.176 & 0.190 \\
0.75 & 0.555 & 0.481 & 0.530 & 0.117 & 0.126 \\
1.00 & 0.643 & 0.564 & 0.621 & 0.0880 & 0.0950 \\
\hline
\end{tabular}

\section{Concluding remarks}

Our main results in this paper, Theorem 1 and Theorem 2, give upper bounds for the probability of ruin of a certain risk process, which (as shown in Section 2) includes as special cases several important models. To obtain Theorem 1 and Theorem 2, first, we obtained an important preliminary result, Lemma 2 , which gave recursive equations for finite-horizon ruin probabilities and an integral equation for the ultimate ruin probability. We illustrated our results with an application to the ruin probability in a risk process with a heavy tail claims distribution, under proportional reinsurance, and a Markov interest rate process. This application suggests that the upper bounds derived by the inductive approach (Theorem 1) are tighter than the upper bounds obtained by supermartingales (Theorem 2). In either case, the upper bounds derived in this paper are sharper than the Lundberg upper bound.

Our paper leaves, of course, many open issues.

(a) Is it possible to obtain bounds tighter than those in Theorems 1 and 2?

(b) Actually, what do we need to obtain the ruin probabilities in closed form? Can we estimate these probabilities using Markov chain techniques (as in [8, pp. 21-39], for instance)?

(c) Can we calculate or estimate quantities such as $\mathrm{E}[\tau]$ or $\mathrm{P}(\tau \leq T)$ for given $T>0$, where $\tau:=\inf \left\{k \geq 1 \mid X_{k}<0\right\}$ is the time of ruin?

These are just a few of the many questions that we can ask ourselves. But two immediate queries are as follows.

(i) Since $\left\{I_{n}\right\}$ in (1) is supposed to be a Markov chain, can we rewrite the minimization of the ruin probability as a Markov decision problem? (See, for instance, [6, pp. 13-42] and [10, pp. VII-XI].) This issue can be settled using the recent results in [5]. We will consider this extension in a forthcoming paper.

(ii) Suppose that in (1) we include an investment process. What can we say about these models?

\section{Acknowledgement}

The authors are grateful to the anonymous referee for his/her constructive comments, which have improved the presentation of the paper.

\section{References}

[1] Asmussen, S (2000). Ruin Probabilities. World Scientific, River Edge, NJ.

[2] CaI, J. (2002). Ruin probabilities with dependent rates of interest. J. Appl. Prob. 39, 312-323. 
[3] CaI, J. And Dickson, D. C. M. (2004). Ruin probabilities with a Markov chain interest model. Insurance Math. Econom. 35, 513-525.

[4] Grandell, J.(1991). Aspects of Risk Theory. Springer, New York.

[5] González-Hernández, J., López-Martínez, R. R. and Pérez-Hernández, J. R. (2007). Markov control processes with randomized discounted cost. Math. Meth. Operat. Res. 65, $27-44$.

[6] Hernández-Lerma, O. and Lasserre, J. B. (1996). Discrete-Time Markov Control Processes (Appl. Math. 30). Springer, New York.

[7] Hernández-Lerma, O. and Lasserre, J. B. (1999). Further Topics on Discrete-Time Markov Control Processes (Appl. Math. 42). Springer, New York.

[8] Hernández-Lerma, O. and Lasserre, J. B. (2003). Markov Chains and Invariant Probabilities (Progress Math. 211). Birkhäuser, Basel.

[9] SснӓL, M. (2004). On discrete-time dynamic programming in insurance: exponential utility and minimizing the ruin probability. Scand. Actuarial J. 3, 189-210.

[10] SchmiduI, H. (2008). Stochastic Control in Insurance. Springer, London.

[11] Willmot, G. E. AND Lin, X. S. (2001). Lundberg Approximations for Compound Distributions with Insurance Applications (Lectures Notes Statist. 156). Springer, New York. 